DE89 012226

\title{
UNDERSTANDING THE PERFORMANCE OF X-RAY MIRRORS
}

Instrumentation Division, Brookhaven National Laboratory

Upton, NY 11973-5000

January 1989
Peter Z. Takacs

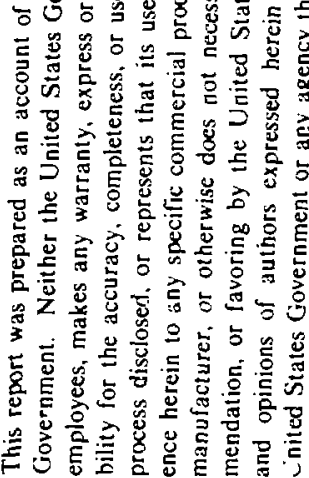

*This research was supported by the U. S. Department of Energy Contract No: DE-AC02-76CH00016. 


\title{
UNDERSTANDING THE PERFORMANCE OF X-RAY MIRRORS
}

\author{
Peter Z. Takacs \\ Instrumentation Division, Brookhaven National Laboratory \\ Upton, NY 11973-5000
}

\section{INTRODUCTION}

The manufacture of $\mathbf{x}$-ray mirrors is a rather specialized branch of the optical fabrication industry. As those who have had to deal with the procurement of these components well know, there are on!y a handful of optical companies who supply most of the grazing incidence optics in use at the synchrotron light source facilities in this country. The reasons for this are several. Firstly, production of a grazing incidence asphere is usually a one-of-a-kind job. It does not lend itself to mass production methods. Not many optical houses are willing to commit the necessary resources for the fabrication of one-of-a-kind components. Secondly, because it is a one-of-a-kind job, it is relatively labor-intensive, which drives up the cost on each individual item. There is a great deal of engineering and planning that must go into the fixturing and design of the computer control program for numerically-controlled diamond turning machines for production of metal optics. Most end users tend to view the procurement of aspheric x-ray optics as a "catalog" process, i.e., that all one needs to do is choose the desired parameters from the manufacturers catalog of stock items and then expect delivery of the finished item (after considerable delay beyond the initial delivery date) which will then perform flawlessly as desired. In reality the procurement process is quite complex, starting with the initial tradeoff studies to choose between metal optics or glass-ceramic optics, continuing with the selection of a vendor who can best do the job, monitoring the progress of the job and ending with the 
final quality assurance acceptance inspection. Many times the component fails to pass the inspection and is returned for further rework. There is relatively little information available of practical use to guide the user through any of the above steps. We have been "forced" to develop our own foundation for assessing the performance of various vendors and determining the quality of the components produced by them. Our approach has been to concentrate on the area of metrology of grazing incidence optics and to develop instruments and techniques that can be used to improve the quality of components delivered to us. The major problem hindering the production of grazing incidence optics is the lack of specialized metrology instrumentation that can be used by the small manufacturing shop to assess the quality of the component under production. We have been engaged over the past several years in developing the theoretical framework and practical measurement techniques to link the metrology to actisal performance, providing much-needed feedback to the manufacturer and also educating users and manufacturers in the proper understanding of the language of surface figure and finish metrology.

\section{THEORETICAL FOUNDATION}

In order to assess the performance of x-ray mirrors in a meaningful way, we must first decide what it is that we need to measure. Certainly we would like to know what the image quality is for light reflected from the mirror surface at the wavelength of operation -- nominally in the 1 to $10 \AA$ range. To do this test at the operating wavelength would be very timeconsuming and would require a very elaborate source and experimental chamber which could accommodate various types and sizes of mirrors with assorted focal parameters. So we are led to ask--"What is it that we can measure on grazing incidence optics?" We would like to measure mirrors off-line, in the laboratory, at visible wavelengths. We would like to measure those characteristics of the surface with visible light that affect the performance of the mirror at $x$-ray wavelengths. The parameters of the surface that meet these criteria are mirror figure 
and finish. We can measure the surface roughness on a microscopic scale and relate the measured quantities to the amount of light scattered from the surface, and we can measure the gross figure errors and relate them to degradation of the image quality.

The terms "figure" and "finish" are rather vague and do not lend themselves to a good quantitative definition. Historically, the division between figure and finish is based upon the types of instrumentation available to make each measurement--conventional interferometry and fringe pattern analysis for figure measurement and visible light-scattering measurements for finish. These methods are totally unsuited for metrology of x-ray optics because conventional interferometry fails at measuring aspheric optics and visible light-scattering probes the surface at spatial periods that are irrelevant to the cause of scattering at $x$-ray wavelengths. The region of surface spatial periods that is most important, from an $x$-ray mirror standpoint, lies between the regions probed by the two conventional methods. Fortunately, over the past few years, commercial insirumentation has been developed that allows one to easily measure the surface finish over the range of spatial periods that is appropriate to near-angle $x$-ray scattering at grazing incidence. ${ }^{1-4}$ These are the spatial periods from several millimeters down to several microns in length. We have employed a microinterferometer system since 1984 to measure surface profiles over a $5 \mathrm{~mm}$ trace length with better than $1 \AA \mathrm{RMS}$ repeatability. ${ }^{5}$ This noncontact method of measuring the surface profile has made it very easy to quantify the quality of full-scale optical components in our laboratory. We have not been so fortunate in the area of figure measurement. No commercial instrument exists that is versatile enough to perform metrology insp...ions on full-size asp̧heric optics with a minimum of set-up time. We have been forced to deveiop our own instrumentation for this task.

But it is not enough to just be able to measure the profile of a surface, one must relate the profile measurements to the desired functional parameters of the mirror under actual operating conditions which are quite different from the normal incidence, visible wavelength metrology conditions. We have devoted considerable theoretical effort over the past several 
years to the development of the tools necessary to make the connection between surface profile measurements and actual performance at $\mathrm{x}$-ray wavelengths. ${ }^{6,7}$ Perhaps the most significant result of our research has been the successful application of the measured surface power spectral density (PSD) function to the prediction of near-angle scattering of $x$-rays from a smooth mirror surface. ${ }^{8}$ The surface PSD function is generated by computing the Fourier transform of individual surface profile measurements and then averaging all Fourier transforms for a given surface. We have shown that the Rayleigh-Rice form of the angle-resolved scattering equation at grazing incidence accurately describes the distribution and intensity of $x$-rays about the specular direction. 'The scattering equation is:

$$
\frac{1}{I_{i}}\left(\frac{d I}{d \theta}\right)_{s}=(1-g) R\left(\theta_{i}\right) \delta\left(\theta_{s}-\theta_{i}\right)+16 \pi^{2}\left(\frac{\sin \theta_{i}}{\lambda}\right)^{3} \cdot R\left(\theta_{i}\right) \cdot K \cdot S(f)
$$

where $S(f)$ is the PSD curve as a function of surface spatial frequency, $f ; R\left(\theta_{i}\right)$ is the Fresnel reflectivity curve at the grazing incidence angle $\theta_{i}$ and $K$ is the materials obliquity factor which depends on the particular theoretical approach used to generate the grazing incidence approximation. The Rayleigh-Rice formalism appears to provide the best fit to the measured scattering data. The form of $K$ for this case is relatively simple:

$$
K=\left(\frac{\theta_{s}}{\theta_{i}}\right]^{2}\left[\frac{R\left(\theta_{s}\right)}{R\left(\theta_{i}\right)}\right.
$$

The predictions of this theory have been borne out in a set of angle-resolved $\mathrm{x}$-ray scatter measurements at $1.39 \AA$ wavelength from a smooth Au-coated $\mathrm{SiC}$ surface at several angles of incidence. Figure 1 illustrates a typical measured surface profile for this surface. The average PSD curve over ten random locations on the surface is shown in Fig. 2. The RMS roughness for this surface is about $3 \AA$ over the full bandwidth. The PSD curve is multiplied by the appropriate factors in Eq. (1) for each of the three angles of incidence and the scaled PSD curves are then plotted over the measured $\mathbf{x}$-ray scattering curves in Fig. 3. The fit of the 
theoretical curves based on the PSD function to the measured $x$-ray scattering curves is quite good. The Rayleigh-Rice formalism accurately predicts both the shape and magnitude of the scattered light distribution and successfully predicts the asymmetry in the wings of the scattered light distribution, which is especially noticeable at the most grazing angle of incidence.

\section{PRACTICAL CONSIDERATIONS}

We have demonstrated that we can relate the scattered light distribution at $\mathbf{x}$-ray wavelengths to the surface roughness properties of a mirror surface through the appropriate theoretical framework. The question now is, "How do we apply this information to the procurement of useable grazing incidence optical components?" The answer to this question is not simple or easy. In the ideal world, if the user knew exactly what he needed in terms of the scattered light distribution from each surface, then it would be a relatively straightforward matter to work backwards through the equation to determine the maximum allowable shape and magnitude for the surface PSD curve. As a rule of thumb one can make a zero-order assumption that the PSD curves for glass-ceramics all have the same power-law shape, only the absolute magnitudes are different. The same holds true for the metal surfaces, although the power-law parameters are usually much different for them.

In practice, the user or optical system designer does not usually have a complete description of the optical system to allow him to deduce the required surface roughness spectrum. Only the most sophisticated of design codes include the surface PSD function as a design parameter.' Other factors which need to be considered are the type of detector that will be used (film, ion chamber, photon counter, etc.), whether or not it is an imaging system, what is the minimum required resolution, the required spectral purity for a monochromator system, the source size, the point spread function caused by "figure" errors, etc. All of these factors tend to complicate the specification of optical components. Contributing to the lack of rigor in grazing incidence system design has been that, in the past, it has not been possible 
to perform the required metrology on the mirrors. Mirror quality has been relatively poor and the credibility of the design process has been rather low because the systems have never performed close to their design goals. Designers and users have resorted to simplistic, backof-the-envelope calculations to specify important surface roughness parameters. Seemingly insignificant changes in these parameters often have a large effect in cost of the final product. Our method of PSD function measurement and performance prediction puts the design process on a much more solid foundation.

\section{MIRROR SUPPLIER DATABASE}

Our measurements of surface roughness began in 1984 after acquisition of a WYKO NCP1000 Digital Optical Profiler. ${ }^{1}$ This instrument allows us to measure the surface roughness of full-sized synchrotron mirrors in a non-contact manner, quickly and efficiently. In the several years since, we have compiled a database of surface roughness as a function of manufacturer, material, and figure type. Recognizing that there is an acute lack of practical information available to the grazing incidence community regarding the above parameters, we have compiled our information in a way that should prove useful for future design considerations.

The mirrors in the database are divided into two distinct classes: flats and aspheres. Conspicuously absent from this database are spherical surfaces. The only spherical surfaces measured to date have been for diffraction gratings and the number of samples is extremely small, so they are not included in this compilation. The aspheric surfaces include cylinders, toroids and ellipsoids. As with the figure types, there are two major material classes: metal substrates and glass or ceramic substrates. The only metal material that is included in this database is electroless nickel plate (ENP), usually on an aluminum substrate. The reason for this is that, for synchrotron use, no other metal surface can be polished to the smoothness required of an $x$-ray reflecting surface. The fact that ENP can also be diamond-machined is also an important reason for its use in SR mirror fabrication. 
The list of manufacturers providing mirrors to the NSLS and other synchrotron facilities whose mirrors have been measured in our Metrology Laboratory is presented in Table 1. A summary of measured mirrors arranged by figure and material type is given in Table II with the number of mirrors of each type identified by manufacturer. Of all the mirrors in this table, only the General Optics silicon flats are not real SR mirrors. These are single-crystal silicon wafers and are included to demonstrate the high-quality optical finish that can be applied to this material if one is careful. Conventional silicon wafers for semiconductor applications are usually an order of magnitude worse in surface roughness.

Table II also summarizes the number of mirrors of each type that have been measured on the BNL WYKO profiler. Please note that not all of these mirrors are for use at the NSLS: a significant fraction were sent here for measurement and then shipped elsewhere for use at other loca...ns, in particular SSRL, SRC in Wisconsin and CHESS. Also, some mirrors are entered twice in the statistics, usually a result of measuring before and after a reworking process. If the mirror failed to meet the specifications, it was sent back to the manufacturer and after reworking was returned here for a second measurement. The most striking examples of reworking to be noted later are early fused silica cylinders made by Frank Cooke around 1981-82, prior to the use of the WYKO profiler for surface metrology. Measurements made on these mirrors in 1985 showed that they had a large amount of low frequency ripple, resulting in RMS roughness numbers that ranged from 25 to $85 \AA$. The low frequency ripple with spatial periods in the millimeter range is extremely difficult to detect by any other means. Frank Cooke subsequently changed his lapping process and, as a result, new cylinders and reworked old cylinders now routinely measure at or below the $5 \AA$ RMS level.

The RMS roughness of each mirror in each column of Table II is plotted roughly as a function of time in the diagram in Figs. 4 through 7. Each figure and material combination is plotted on a separate graph to better enable visual comparisons between various permutations of the parameters. The horizontal axis labelled "Volume ID Number" refers to 
the data file identification number in the WYKO profiler log book. It is basically a time series with Volume 1 starting in 1984 and Volume 5 starting in January 1988 covering the first half of 1988 . The data points refer to the date on which the mirror was measured in the laboratory, not necessarily to the date the mirror was manufactured. In fact, some mirrors that were manufactured many years ago have only recently been measured. Most measurements in this database were made with the $2.5 \mathrm{X}$ magnification objective on our WYKO instrument. Only a few of the early measurements were made with the $10 \mathrm{X}$ objective. The $2.5 \mathrm{X}$ objective is now used almost exclusively, since it provides significant information about the roughness in the important spatial period region from 1 to 5 millimeters. This is the region that is most inaccessible to other metrology techniques and is what makes our measurement capability unique. The roughness numbers plotted are for the full $2.5 \mathrm{X}$ bandwidth which covers the range from 5.0 millimeters down to 9.8 micrometers.

Figure 4 summarizes the results for glass/ceramic aspheres. The three types of aspheres measured are cylinders, ellipsoids, and toroids. Most of the mirrors are made from fused silica; a few are ZERODUR, two are single-crystal silicon and two are CVD silicon carbide. Some are coated with a metal reflecting layer, some are uncoated. We almost never see evidence that a properly-applied thin metal coating changes the roughness properties of the surface, although occasionally a bad coating does slip in.

The majority of glass/ceramic aspheres have been right circular cylinder segments, primarily used for sagittal focusing of a horizontal fan of synchrotron radiation. The group of 5 cylinders located in a vertical column at " $A^{n}$ in Fig. 4 were manufactured a few years earlier by Frank Cooke. As mentioned above, they were only first measured at the time indicated in the graph. Several other Frank Cooke cylinders with RMS roughness values above $20 \AA$ were also manufactured in this early time frame, but were only recently measured. Those whose early manufacture date could be confirmed are indicated with an arrow pointing to earlier times. The major trend to note is the significant reduction in the average RMS roughness of 
the cylinders in more recent times. The early cylinders were usually always above 20 \& RMS, while now it is unusual to see one that exceeds $10 \AA$ RMS. A dramatic example of the improvement in the polishing process based on feedback from the metrology is indicated by the data points marked with asterisks. An early fused silica mirror with a roughness of about 40 A RMS was reworked and measured in 1988 to have an RMS roughness slightly more than 3 A. Another example of improved surface finish is the series of three measurements on a cylinder at "B" connected by the straight lines. The initial measurement was $23.3 \AA$ RMS. Additional work on the surface produced a $25 \AA$ surface (not statistically different from the first measurement), but additional work and a process change produced a surface with a 7.8 A RMS finish. The mirror at $\mathrm{C}$ was originally made years ago, but after reworking its roughness level decreased from $83 \AA$ to $5.89 \AA$ RMS. A subsequent platinum coating was measured at 5.4 \& RMS, again not statistically different from the uncoated surface. Occasionally, however, a new mirror slips through with a very rough surface, as indicated by the point labeled " $\mathrm{D}$ " at about $70 \AA \mathrm{RMS}$ in the upper right corner. This illustrates the usefulness in being able to make these kinds of measurements on a regular basis. Other products in this class from other vendors all lie below the $10 \mathrm{~A}$ level, which differs significantly from the metal mirror results.

The data for the metal aspheres is plotted in Fig. 5. One can see almost immediately that there appears to be a lower limit of about $10 \AA$ RMS to the best that can be achieved on this material, independent of figure. The best surface to date in the electroless nickel material is the most recent product from Optic-Electronic Corp., a cylinder with an RMS roughness of $8.45 \AA$, but whether or not this low value can be improved upon remains an open question. All the evidence indicates that it is very difficult to produce a surface below the $10 \AA$ level with ENP, while for glass/ceramic materials it is highly unusual to exceed the $10 \AA$ level. Pairs of data points in Fig. 5 also indicate the improvement in surface finish of a set of ellipsoidal mirrors obtained after reworking the mirrors. In each case the rework involved additional 
polishing on the surfaces. The pair of data points above the $100 \mathrm{~A}$ level are for a rather complex ellipsoid designed to produce an 8 to 1 image size reduction. ${ }^{10}$ The numerical machining control problem was extremely difficult to solve to produce the desired contours with 3 degrees of freedom in the machine motion. Additional polishing was not able to remove the low frequencies left in the surface. The set of open squares labeled " $A$ " indicate before and after measurements on two ENP ellipsoids that were coated with gold-electroplate. Again, no change in the roughness was evident.

More compelling evidence for the $10 \AA$ roughness barrier can be seen by comparing the glass/ceramic flats in Fig. 6 with the ENP flats in Fig. 7. The two silicon carbide flats above $10 \AA$ are really grating test samples and should not be included in the comparison. The only other flat above $10 \AA$ is a piece of float glass with the unusual property of being very smooth along the pull direction, but with a well-defined, large amplitude sinusoidal low-frequency period in the transverse direction. A vertical line connects the two directions on the graph. Most of these flats are intended to be used in mirror benders to produce focusing in the tangential direction and, in the case of specially-configured cross-section pieces, focusing in the sagittal direction also. The ENP flats in Fig. 7 again show a distinct cutoff at the $10 \AA$ level. There are none better than $8 \AA$. The only example of degradation in surface roughness after coating is seen at the right-hand side of Fig. 7. A thick platinum coating was evaporated onto a $70 \mathrm{~cm}$ long ENP mirror and the surface roughness increased from $15 \AA$ before to $35 \AA$ after coating.

\section{DISCUSSION}

The division line at $10 \AA$ between the ENP and glass/ceramic materials appears to be independent of figure type. The same division is seen in the data from the aspheres as from the flats. In fact, the best finishes for each material appear to be independent of figure type. One can achieve the same quality finish on aspheres as on flats, so there should be no need to relax finish performance requirements for non-flat surfaces. Aspheric surfaces are, however, 
more difficult to produce than are flats, especially when one desires a multiaxis machine cut, as with toroids and ellipsoids. Cylinders can be easily manufactured both in glass/ceramic and metal materials because only one uniform radius needs to be generated in the surface. Highe:order aspheres can be made more easily in metals than in glass, and subsequently for less cost per piece, but the performance may suffer because of the limits on polishability. The electroless rickel plating process is subject to many factors that are usually beyond the control of the mirror fabricator. There are many variables that go into the ENP process that are not controlled in an ordinary industrial plating operation. Mirror manufacturers often report that they had to have the ENP coating stripped and replated several times before the final diamond-machined and polished surface met the specifications. These iterations tend to drive the price up on metal mirrors. Very few manufacturers can adequately characterize the quality of the ENP coating before polishing the piece. These considerations are non-existent for glass/ceramic materials, except for the case of silicon carbide. A layer of chemical vapor deposited (CVD) SiC is deposited on the surface of another substrate material and the CVD layer is then polished to the desired finist. Like the ENP process, the CVD process is also subject to many variables which are often difficult to control. A different set of problems arise in polishing the $\mathrm{SiC}$, but the end result could be the same: the need to redeposit or replate more material and polish again.

Each material has its advantages and its drawbacks. Metal mirrors allow designers more flexibility in terms of optical design by permitting complex surfaces to be machined, but at the present time the price is poor surface finish. Glass/ceramic flats are easily made, but their usefulness in imaging is not very great unless they are bent. Perha as one will need to spend more effort in engineering the cross-sectional shape of a flat mirror and in the design of the bending mechanism to produce the desired tangential and sagittal curvatures from a single piece of float glass, instead of passing on the complexity to the optical fabricator to make an aspheric surface. The bent-mirror solution may not work in all cases, especially those involving 
actively-cooled mirrors. The trend in optical design for higher-brightness source instrumentation appears to be away from aspheric optics and towards spherical optics used at grazing incidence. The consensus is that spherical surfaces can be made more easily to meet the strici figure tolerances required by newer SR sources than can asṇheric surfaces. However, careful consideration must be given to choice of substrate material in order not to compromise the scattered light performance of the system.

\section{ACKNOWLEDGMENT}

I would like to acknowledge the assistance of Dr. Eugene L. Church in providing the theoretical framework for understanding the relationship between surface roughness and scattered light distribution at grazing incidence. This research was supported by the $U$. S. Department of Energy: Contract No. DE-AC02-76CH00016.

\section{REFERENCES}

1. J. C. Wyant, C. L. Koliopoulos, B. Bushan, and O.E. George, An Optical Profilometer for Surface Characterization of Magnctic Media, ASLE Trans 그, 101 (1984).

2. J. M. Eastman and J. M. Zavislan, A New Optical Surface Microprofiling Instrument, Proc. SPIE 429 (1983).

3. G. E. Sommargren, Optical Heterodyne Profilometry, Appl. Optics 20, 610 (1981).

4. J. F. Biegen and R. A. Smythe, High Resolution Phase Measuring Laser Interferometric Microscope for Engineering Surface Metrology, in: "Scanning Microscopy Technologies and



5. E. L. Church and P. Z. Takacs, Use of an Optical-profiling Instrument for the Measurement of the Figure and Finish of Optical-quality Surfaces, WEAR 109, 241-257 (1986).

6. E. L. Church, H. A. Jenkinscn, and J. M. Zavada, Relationship Between Surface Scattering and Microtopographic Features, Opt. Eng. 18125 (1979). 
7. E. L. Church and P. Z. Takacs, The Interpretation of Glancing Incidence Scattering Measurements, Proc. SPIE 640, 126 (1986).

8. In preparation for submittal to Applied Optics.

9. Pau! Glenn, Space Telescope Performance Prediction Using the OSAC Code, in: "Large Optics Technology," Proc. SPIE $\underline{\text { 771, }}$ 164-179 (1985).

10. K. W. Jones, P. Z. Takacs, J. B. Hastings, J. M. Casstevens, and C. Pionke, Fabrication of an 8:1 Ellipsoidal Mirror for a Synchrotron X-ray Microprobe, in: "Metrology: Figure and Finish," Bruce Truax, ed., Proc. SPIE 749, $37-44$ (1987). 
TABLE I. Suppliers of Synchrotron Radiation Mirrors Included In This Survey.

American Aspheric Co.

Applied Optics Center Corp.*

Continental Optical Corp.

Diamcnd Electro-Optics, Inc.

Farranti Astron, Ltd.

Frank Cooke, Inc.

General Optics

Karl Lambrecht Corporation

Liberty Mirror

Libbey-Owens-Ford

Optic-Electronic Corporation
P.O. Box 22303

Tucson, AZ 85734

Burlington, MA

15 Power Drive

Hauppauge, NY 11787

323 Andover Street

Wilmington, MA 01887

Unit 1, Aerodrome Way

Cranford Lane

Hounslow TW5 9QB

UNITED KJNGDOM

59 Summer Street

North Brookfield, MA 01535

554 Finn Avenue

Moorpark, CA 93021

4204 N. Lincoln Avenue

Chicago, IL 60618

851 Third Avenue

Brackenridge, PA 15014

11545 Pagemill Road

P.O. Box 740668

Dallas, TX 75374-0668

Waltham, MA

Oak Ridge, TN

Union Carbide Nuclear Division

Y12 Plant

(Now under management by Martin Marietta Corporation)

*The mirror fabrication facility from this organization is now a part of Diamond ElectroOptics. 
$-15-$

TABLE II. Synchrotron Radiation Mirrors Measured for Surface Roughness

\begin{tabular}{|c|c|c|c|c|c|c|c|c|}
\hline \multirow{3}{*}{ Figure } & \multicolumn{4}{|c|}{ ASPHERES } & \multicolumn{4}{|c|}{ FLATS } \\
\hline & \multicolumn{4}{|c|}{ Material } & \multicolumn{4}{|c|}{ Material } \\
\hline & ENP/Al & Gla & Ceramic & & ENP/A] & & Glass, Ceramic & \\
\hline Cylinder & $\begin{array}{l}\text { OEC } \\
\text { ROD } \\
\text { Astron }\end{array}$ & $\begin{array}{l}4 \\
1 \\
2\end{array}$ & $\begin{array}{l}\text { F. Cooke } \\
\text { Continental } \\
\text { Am. Asph. }\end{array}$ & $\begin{array}{r}24 \\
5 \\
1\end{array}$ & $\begin{array}{l}\text { OEC } \\
\text { AOC } \\
\text { ROD } \\
\text { DEO }\end{array}$ & $\begin{array}{l}3 \\
1 \\
5 \\
2\end{array}$ & $\begin{array}{l}\text { Liberty } \\
\text { F. Cooke } \\
\text { Continental } \\
\text { Continental }\end{array}$ & $\begin{array}{ll}5 & \text { (Float G) } \\
4 & \text { (FS) } \\
1 & \text { (FS) } \\
4 & \text { (SiC) }\end{array}$ \\
\hline Ellipsoid & $\begin{array}{l}\text { DEO } \\
\text { AOC } \\
\text { Unknown } \\
\text { OEC }\end{array}$ & $\begin{array}{r}18 \\
1 \\
2 \\
2\end{array}$ & $\begin{array}{l}\text { Continental } \\
\text { Unknown }\end{array}$ & $\begin{array}{l}1 \\
1\end{array}$ & $\begin{array}{l}\text { Astron } \\
\text { Y12 }\end{array}$ & $\begin{array}{l}2 \\
1\end{array}$ & $\begin{array}{l}\text { Karl L } \\
\text { Astron } \\
\text { Genl. Opt. }\end{array}$ & $\begin{array}{ll}4 & \text { (Float G) } \\
2 & \text { (SiC) } \\
4 & \text { (Si) }\end{array}$ \\
\hline Toroid & $\begin{array}{l}\text { Astron } \\
\text { AOC } \\
\text { OEC }\end{array}$ & $\begin{array}{l}1 \\
1 \\
1\end{array}$ & $\begin{array}{l}\text { Am. Asph. } \\
\text { Astron }\end{array}$ & $\begin{array}{l}1 \\
6\end{array}$ & & & & \\
\hline $\begin{array}{l}\text { Total Measurements } \\
\text { Per Category: }\end{array}$ & & 3 & & 36 & & 14 & & 24 \\
\hline
\end{tabular}




\section{FIGURE CAPTIONS}

Figure 1. A typical surface profile for a smooth Au-coated $\mathrm{SiC}$ mirror measured with a WYKO NCP-1000 Digital Optical Profiler with a $2.5 \times$ magnification objective.

Figure 2. The power spectral density (PSD) curve for the Au-coated SiC surface, generated by averaging the PSD curves from 10 random locations on the surface. The RMS surface roughness for this surface, computed by integrating the area under the PSD curve, is $3.1 \AA$ over the full measurement width.

Figure 3. Predicted and measured scattered light distributions for the Au-coated surface at a wavelength of $1.39 \AA$ at three angles of incidence. The measured scattered light curves (smooth lines) are normalized to the incident beam intensity: the relative intensity of the specular peak is given by the Fresnel reflectivity of the surface for each particular angle of incidence. The predicted scattered $\mathrm{x}$-ray distributions (rough lines) computed from Eqs. (1) and (2) are overlayed on the appropriate measured curve. The agreement in the overall shape and magnitude of the Rayleigh-Rice formalism is quite good. The theory correctly predicts the asymmetry between the two sides of the scattered light distribution.

Figure 4 Measured RMS roughness values plotted as a function of laboratory logbook volume and page number (roughly a time sequence starting in 1984 and ending in early 1988). This is not a time sequence of when each mirror was manufactured. Those marked with the left-pointing arrows were manufactured before 1984. See text for full explanation. The diagram illustrates that fabrication techniques for glass and ceramic aspheres are good encugh to routinely produce surfaces with roughness values well below $10 \AA$ RMS.

Figure 5. Measured RMS roughness values for aspheres made from electroless nickel plate (ENP) on an aluminum substrate. The solid lines connect measurements made on the same mirror before and after repolishing. The diagram illustrates the difficulty in achieving RMS roughness values below $10 \AA$ with this material. 
Figure 6. Measured RMS roughness values for flat surfaces made from glass or ceramic materials. The solid vertical line connects measurements on a piece of float glass that shows an extreme anisotropy in surface roughness between the direction of pull (smooth) and the transverse direction (large amplitude long-period sinusoid). Most measurements lie well below the $10 \AA$ RMS level.

Figure 7. Measured RMS roughness values for flat surfaces made from ENP/AI material. Most values lie above the $10 \AA$ level. The solid line shows the effect of a poor coating on a previously acceptable surface. 


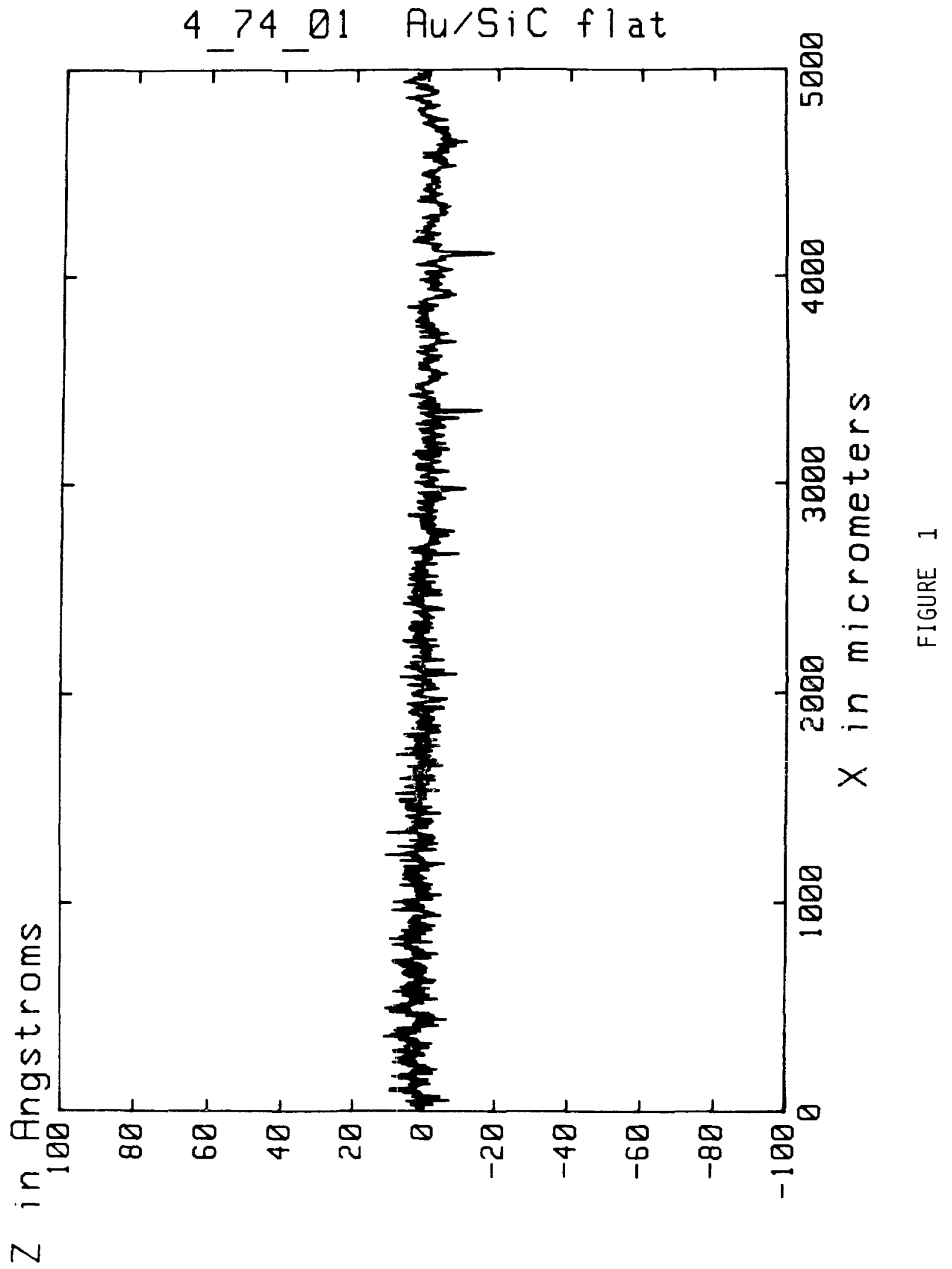




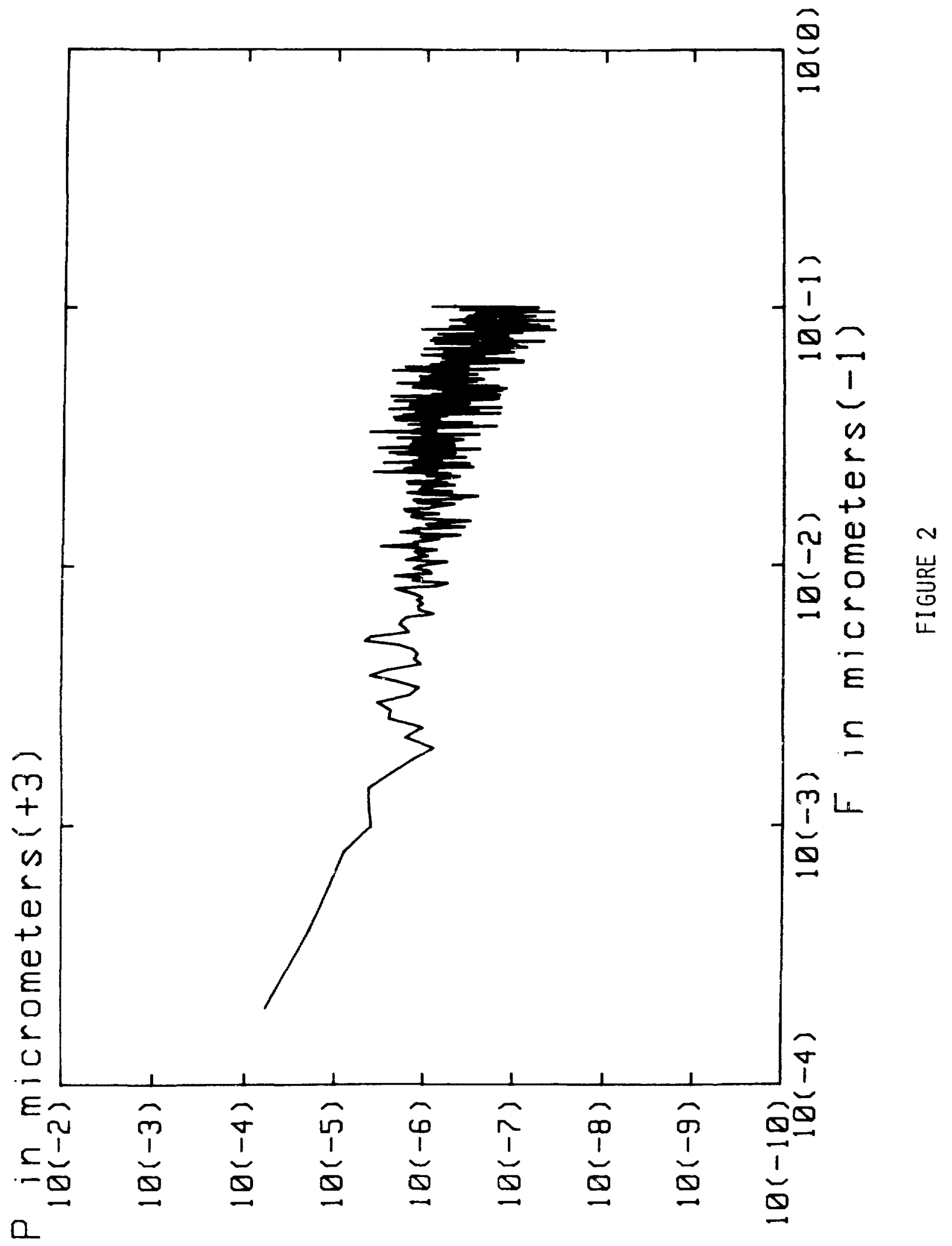




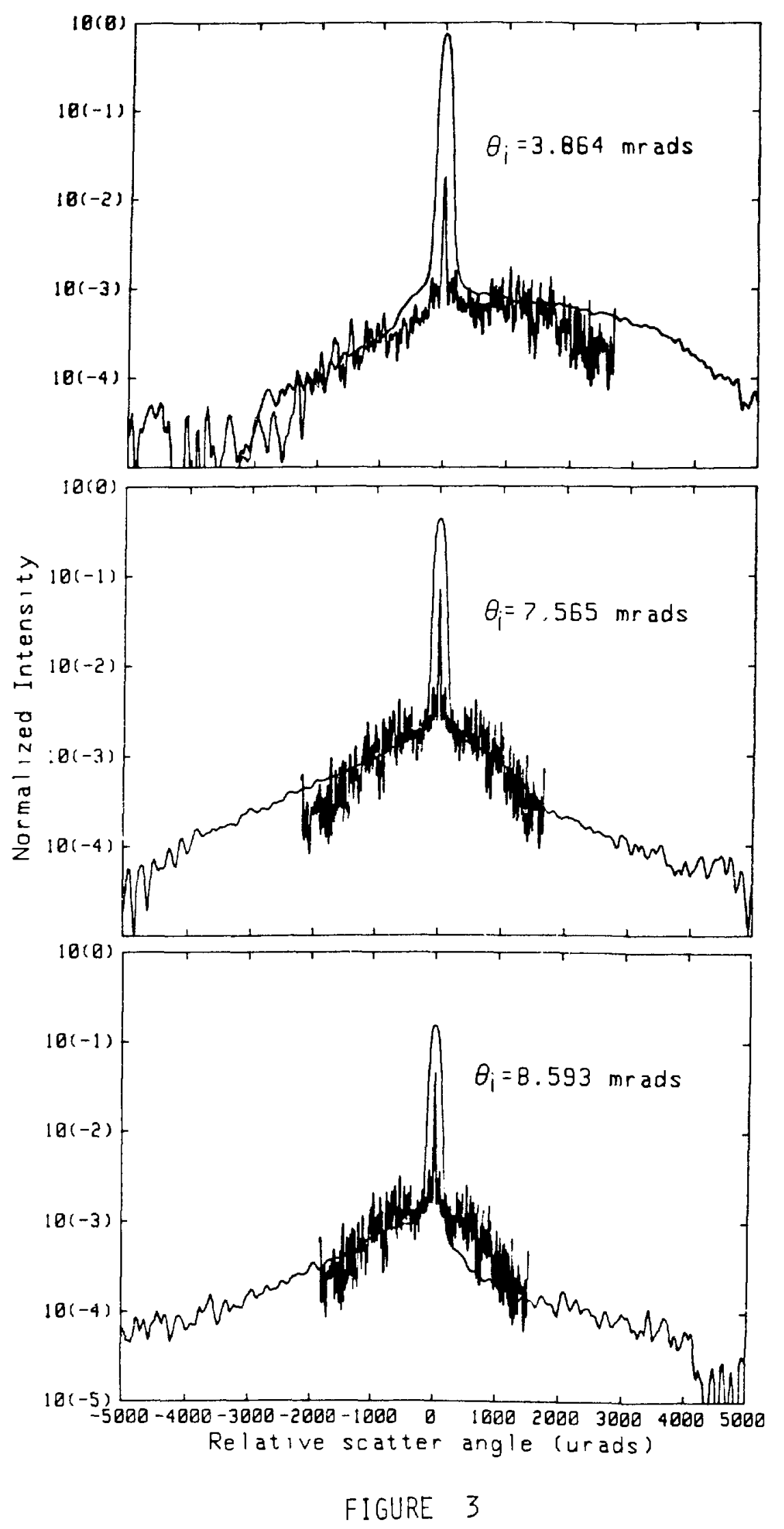









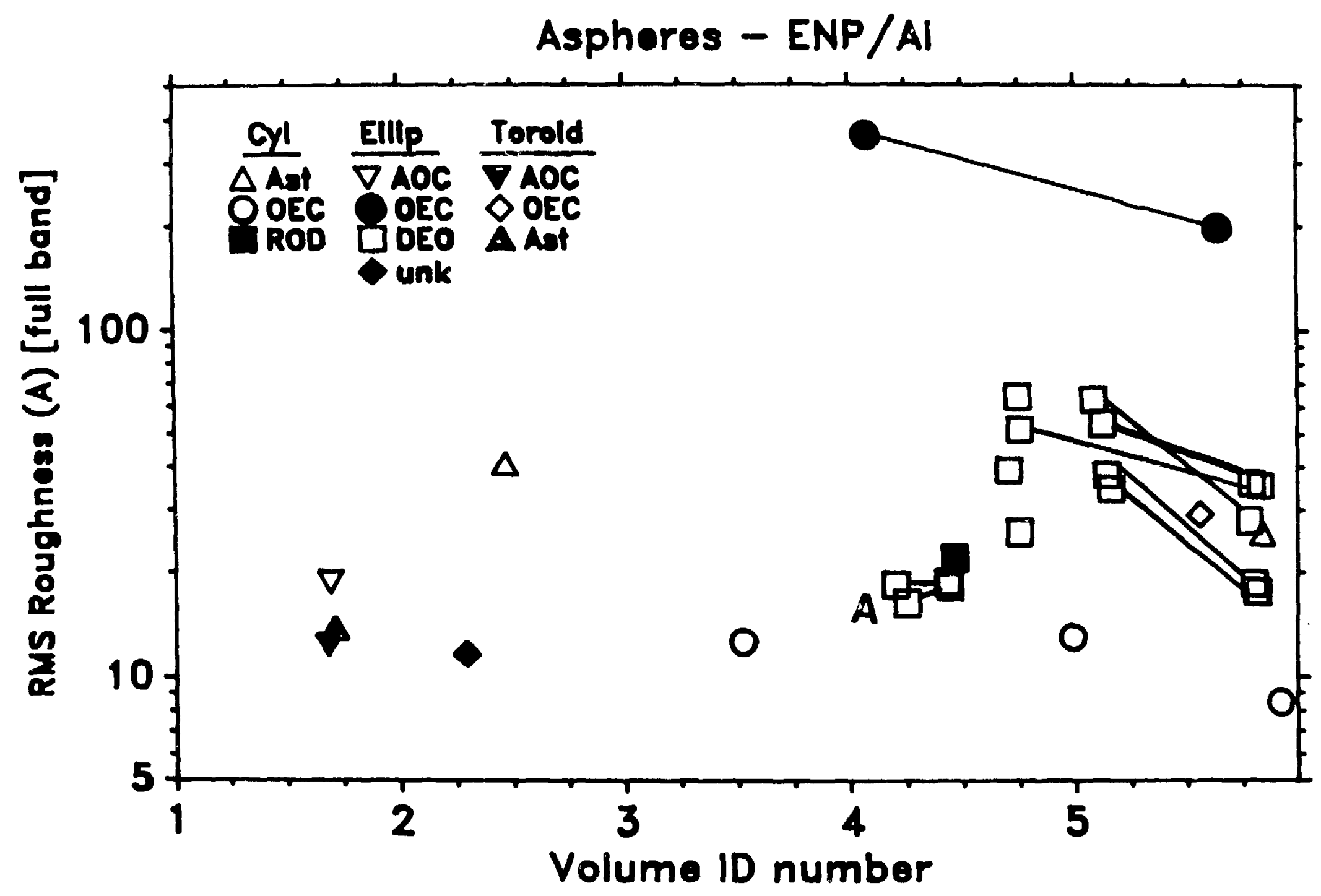

FIGURE 5 
Flats - Glass,Ceramic

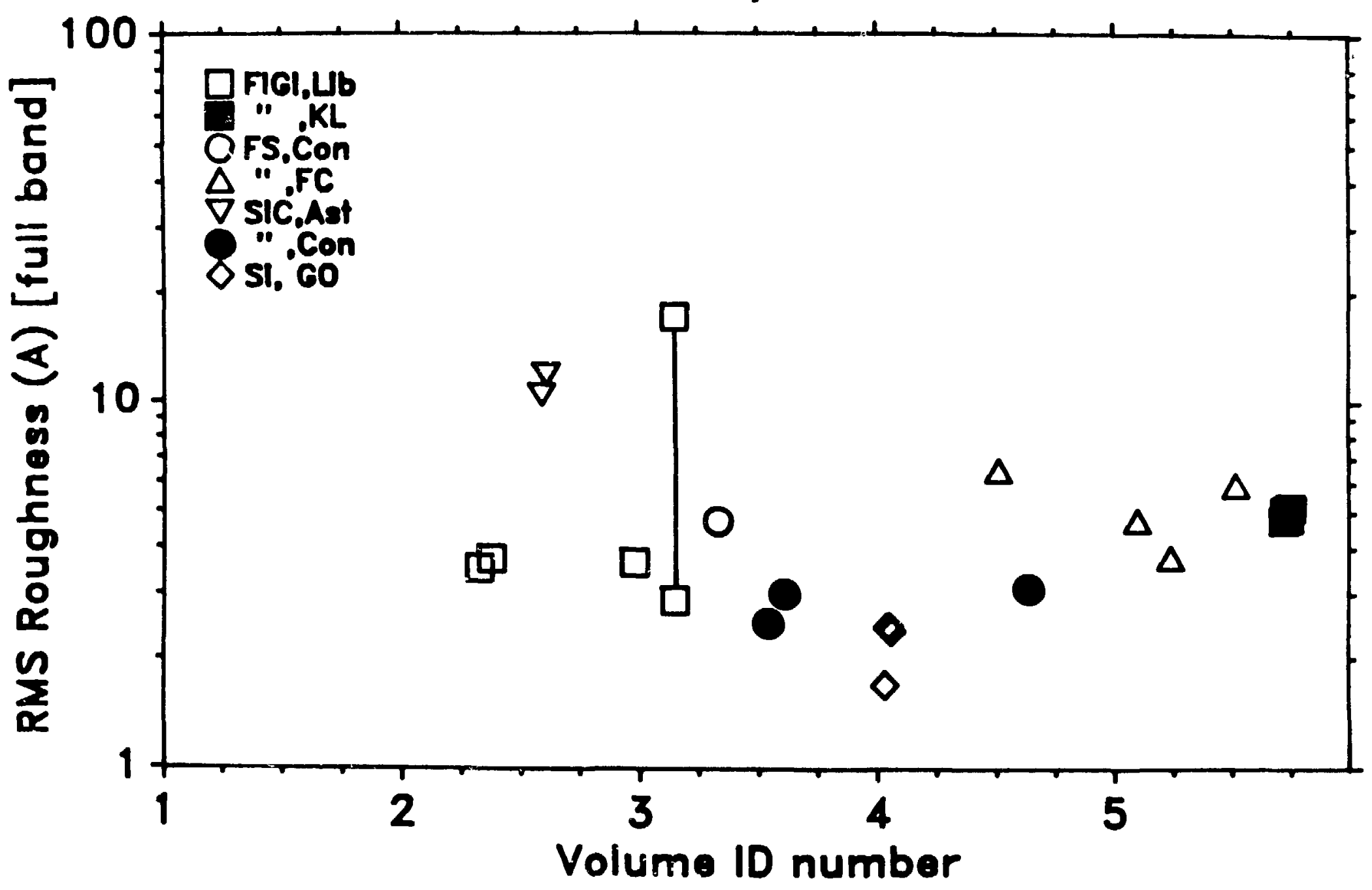


Flats - ENP/Al

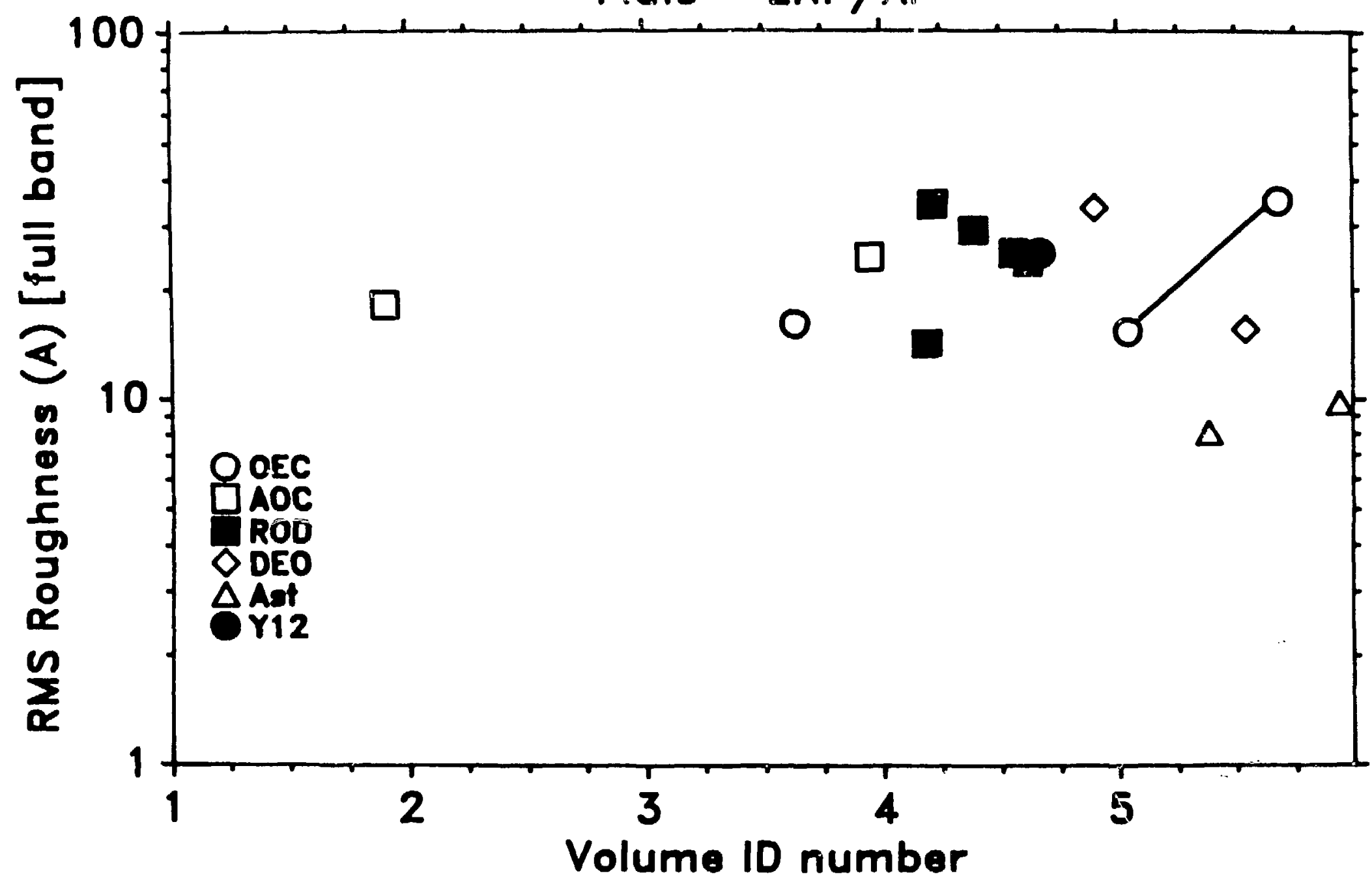

